Original paper

\title{
Automatic VMAT planning for post-operative prostate cancer cases using particle swarm optimization: A proof of concept study
}

\author{
Luise A. Künzel $^{\mathrm{a}, *}$, Sara Leibfarth ${ }^{\mathrm{a}}$, Oliver S. Dohm ${ }^{\mathrm{b}}$, Arndt-Christian Müller ${ }^{\mathrm{b}}$, Daniel Zips ${ }^{\mathrm{b}, \mathrm{c}}$, \\ Daniela Thorwarth ${ }^{\text {a,c }}$ \\ a Section for Biomedical Physic, Department for Radiation Oncology, University Hospital Tübingen, Hoppe-Seyler-Str. 3, 72076 Tübingen, Germany \\ ${ }^{\mathrm{b}}$ Department for Radiation Oncology, University Hospital Tübingen, University Hospital Tübingen, Hoppe-Seyler-Str. 3, 72076 Tübingen, Germany \\ c German Cancer Consortium (DKTK), Partner Site Tübingen; and German Cancer Research Center (DKFZ), Heidelberg, Germany
}

\section{A R T I C L E I N F O}

\section{Keywords:}

Radiotherapy

Automatic planning

Particle swarm optimization

VMAT

\begin{abstract}
A B S T R A C T
Objective: To investigate the potential of Particle Swarm Optimization (PSO) for fully automatic VMAT radiotherapy (RT) treatment planning.

Material and Methods: In PSO a solution space of planning constraints is searched for the best possible RT plan in an iterative, statistical method, optimizing a population of candidate solutions. To identify the best candidate solution and for final evaluation a plan quality score (PQS), based on dose volume histogram (DVH) parameters, was introduced.

Automatic PSO-based RT planning was used for $N=10$ postoperative prostate cancer cases, retrospectively taken from our clinical database, with a prescribed dose of $E U D=66 \mathrm{~Gy}$ in addition to two constraints for rectum and one for bladder. Resulting PSO-based plans were compared dosimetrically to manually generated VMAT plans.

Results: PSO successfully proposed treatment plans comparable to manually optimized ones in 9/10 cases. The median (range) PTV EUD was 65.4 Gy (64.7-66.0) for manual and 65.3 Gy (62.5-65.5) for PSO plans, respectively. However PSO plans achieved significantly lower doses in rectum $D_{2 \%} 67.0$ Gy (66.5-67.5) vs. 66.1 Gy (64.7-66.5, p = 0.016). All other evaluated parameters (PTV $D_{98 \%}$ and $D_{2 \%}$, rectum $V_{40 G y}$ and $V_{60 G y}$, bladder $D_{2 \%}$ and $V_{60 G y}$ ) were comparable in both plans. Manual plans had lower PQS compared to PSO plans with -0.82 ( $-16.43-1.08)$ vs. $0.91(-5.98-6.25)$.

Conclusion: PSO allows for fully automatic generation of VMAT plans with plan quality comparable to manually optimized plans. However, before clinical implementation further research is needed concerning further adaptation of PSO-specific parameters and the refinement of the PQS.
\end{abstract}

\section{Introduction}

Modern medical linear accelerators (Linacs) as used for today's cancer treatments with radiotherapy (RT) offer an enormous amount of degrees of freedom with respect to the technical possibilities when it comes to treatment plan application and delivery. To make use of those opportunities modern RT treatment planning is formulated as an inverse optimization problem in order to achieve maximum dose coverage in the tumor volume and at the same time a minimum of dose burden to organs at risk (OAR) and healthy tissues [1-3]. Solving this optimization problem may be considered trivial, but the correct formulation of this problem in order to achieve the best possible RT plan for an individual patient is extremely challenging. The problem formulation contains dose constraints and objectives regarding to tumor and organ at risk (OAR) doses [3]. The challenge here is to balance all presets to ensure the coverage of the tumor with the prescribed dose and at the same time achieve the best possible dose sparing for OARs. In clinical practice planners alter those constraints to find an appropriate plan. Thus, RT planning is often a time consuming trial and error process, which is not reproducible and its success depends highly on the planner's experience. Automation of the planning process may overcome those problems and therefore automatic treatment planning gained attention in recent years [4-10]. In principle, three major conceptual approaches have been proposed for automatic RT planning in the last years. On the one hand dose mimicking approaches were investigated [11-14], they basically rely on an atlas of previously treated

\footnotetext{
* Corresponding author at: Hoppe-Seyler-Str. 3, 72076 Tübingen, Germany.

E-mail address: luise.kuenzel@med.uni-tuebingen.de (L.A. Künzel).
} 
patients and the plan for the actual patient is calculated by using information from the most similar plan in the atlas. On the other side template-based approaches seem to be very promising [15-18], which try to formulate the optimization problem as accurate as possible for each individual patient. A third, recently introduced concept uses deep learning to predict the dose distribution and then convert this estimated distribution into optimization objectives [19-21]. However, a major problem for all automatic planning approaches is how to objectively compare the different plans. There are attempts to design scores like the Plan Quality Metric [22] proposed by Nelms et al. to compare plans in an inter-institutional planning study or a combination of a graphical representation of planning objectives combined with a score index to support clinicians in their decision [23] proposed by Ventura et al. Nevertheless the design of such scores is difficult and by now they are not widely used in clinical routine.

We propose to treat the formulation of the aforementioned optimization problem, which may also be seen as fine-tuning of a template, as a second optimization problem, and hence to solve it by an appropriate optimization algorithm. Our hypothesis is that Particle Swarm Optimization (PSO), which was first introduced by Kennedy and Eberhart in 1995 [24] as a statistical optimization for non-linear functions, can be used for automatic RT planning. As the intensity modulated radiotherapy (IMRT) problem is of non-linear nature and of high dimensionality, PSO seems to be a promising solution. PSO is under active research and widely used in different fields, such as medical engineering [25] and logistics [26]. PSO was also considered for radiotherapy treatment planning already: Li et al. proposed to use it for beam-angle-selection in IMRT [27], whereas Riofrio et al. used PSO for high-dose-rate brachytherapy planning [28]. An approach for inverse planning of four dimensional conformal lung stereotactic body radiation therapy (SBRT) was proposed by Modiri et al. [29,30] and Yang et al. introduced PSO to automatically select the optimization weights for IMRT planning [31].

The principle idea of PSO is to exchange and combine the information gained by individuals, named particles, about their quality to solve complex problems. Therefore the quality of a treatment plan has to be measurable, which was realized in this study by introducing a plan quality score (PQS), similar to the aforementioned scores in previously published plan comparison [22,23]. In order to realize automatic volumetric modulated arc therapy (VMAT) treatment planning, we propose here to start from a treatment specific template of planning constraints. The template contains the types of constraints, but the appropriate planning constraint value is patient specific and therefore unknown. This is similar to manual planning where these planning constraints are set in a time consuming trial and error process. In contrast to a manual planner, PSO is expected to be reproducible and more consistent and eliminates the subjective influence of a human planner. PSO is linked to the treatment planning system (TPS) to balance the planning constraint values in such a way, that the OAR constraints are adhered to and at the same time the prescribed tumor dose is reached. Therefore, our hypothesis is that PSO has the potential to substitute manual trial and error-based treatment planning and may hence be a potential solution for automatic planning.

\section{Material and Methods}

\subsection{Constrained optimization}

A dose distribution can be mathematically defined as vectoral sum of dose effects regarding targets $\left(F_{i}\right)$ and OARs $\left(G_{j}\right)[3]$ :

$\left(F_{1}, \cdots F_{n}, G_{1}, \cdots G_{m}\right) \in\left[0, \infty\left[{ }^{n+m}\right.\right.$

In principle every combination of dose effects would be possible, but not all are practically applicable nor clinically favorable. A clinically favorable distribution should cover the target with dose, while sparing OARs from dose burden. Moreover, a plan where no target dose or any
OAR sparing can be improved without deteriorating another one is preferential, which is the definition of pareto-optimality. In the TPS Hyperion a constrained optimization based on the Lagrangian function $L$ is used to define a pareto-optimal and clinically favorable plan [3]:

$L=\sum_{i=1}^{n} F_{i}+\sum_{j=1}^{m} \lambda_{j}\left(G_{j}-C_{j}\right)$

$C_{j}$ determine the constraints and $\lambda_{j}$ the corresponding Lagrangian multipliers. A clinically favorable plan is chosen by defining a patient individual set of constraints $C_{j}$ where the constraints describe the maximum accepted dose effect to the OARs. If possible without deteriorating target objectives, dose effects $G_{j}$ will even be pushed below the constraints $C_{j}$, allowing for a better OAR sparing compared to the predefined dose allowance $C_{j}$. As this is the definition of pareto-optimality, plans generated by the TPS Hyperion are considered paretooptimal [3]. This is ensured by the barrier-penalty multiplier method used to solve the constrained optimization problem, for detailed information we refer to [32]. Consequently, running an optimization with non-binding constraints yields a solid estimation of the minimum possible OAR sparing. Therefore, the related dose effects $G_{j}$ ( $c f$. equation (2)) can be obtained from the TPS. Nevertheless, the selection of constraints, i.e. assessed dose effects, has to reflect clinical needs in order that a pareto-optimal plan equals a clinically optimal plan.

TPS Hyperion allows for the usage of biological dose constraints, $c f$. [3] for detailed information. In this study serial dose constraints based on the concept of generalized gEUD (equivalent uniform dose) [33] were used:

gEUD $=\left(\sum_{i=1}^{N} v_{i} D_{i}^{a}\right)^{\frac{1}{a}}$

where $v_{i}$ and $D_{i}$ are volume and dose bins from the DVH and " $a$ " is a constraint specific parameter. For $a=1$ EUD equals mean dose ( $\left.\mathrm{D}_{\text {Mean }}\right)$ and with increasing " $a$ " EUD is steered towards the maximum dose. Therefore serial constraints can be used to control different regions of DVH for the same structure. TPS Hyperion receives the allowed gEUD as constraint.

\subsection{Particle swarm optimization}

PSO is a nature-analogous, collective, iterative and statistical optimization for non-linear problems. The basic idea of PSO is to simulate the relatively simple behavior of individuals in nature, such as fish or birds, which form swarms of hundreds or thousands to solve complex problems by sharing information, e.g. search for food. To adopt this idea, PSO needs to meet four prerequisites:

I. Each particle knows its own position in the search space $\left(x_{i, d}\right)$. II. Particles are able to assess the quality of their position (fitness).

III. Each individual memorizes the best position reached so far $\left(p_{\text {best }, i}\right)$ and the corresponding fitness value.

IV. Particles communicate to share their best positions and can thereby identify the overall best position $\left(g_{\text {best }}\right)$.

This information is then used to update each particle's position $x_{i}$ in the next iteration $d$ :

$x_{i, d}=x_{i, d-1}+\Delta x_{i, d}$

where the $\Delta x_{i}$ denominates the relocation of each particle. The relocation $\Delta x_{i}$ is calculated by a sum of three different components:

$\Delta x_{i, d}=\omega * \Delta x_{i, d-1}+c_{1} * \zeta *\left(p_{\text {best }, i}-x_{i, d-1}\right)+c_{2} * \xi *\left(g_{\text {best }}-x_{i, d-1}\right)$

Here, the first summand is called nostalgia or inertia and is derived from the previous relocation vector $\Delta x_{i, d-1}$ and the inertia weight $\omega$. The inertia weight $\omega$ was first introduced in 1998 by Shi and Eberhart [34] 
Table 1

Summary of PSO parameter symbols, meaning with related explanations for radiotherapy treatment planning and parameter values.

\begin{tabular}{|c|c|c|c|}
\hline Symbol & Meaning & Explanation & Parameter values \\
\hline$I$ & Number of particles & $\begin{array}{l}\text { A particle is a set of planning constraints, translating to a } \\
\text { plan/dose distribution }\end{array}$ & $I=30$ \\
\hline$N$ & Dimensionality of the solution space & Identical to number of planning constraints & $N=3$ \\
\hline$D$ & Number of generations & Number of PSO iterations & $D_{\text {Max }}=100$ \\
\hline$\zeta, \xi$ & Vectors of random numbers & $\begin{array}{l}N \text {-dimensional vectors, with components each sampled from } \\
\text { the interval }[0,1]\end{array}$ & from interval $[0,1]$ \\
\hline$c_{1}, c_{2}$ & Optimization constants & $\begin{array}{l}\text { To balance relocations, these constants are held fixed during } \\
\text { whole optimization process }\end{array}$ & $c_{1}=c_{2}=2[24]$ \\
\hline$\omega$ & inertia weight & $\begin{array}{l}\text { Balances exploration and exploitation. This parameter is } \\
\text { adapted during optimization process. }\end{array}$ & $\begin{array}{l}\omega=1.4 \text { in generation } 0-9 \text {, reduced by } 0.2 \text { every } 10 \text { th generation } \\
\text { until } 0.2 \text { is reached in generation } 50\end{array}$ \\
\hline$x_{i, d}$ & $\begin{array}{l}\text { Current position of particle } i \text { in } \\
\text { generation } d\end{array}$ & $\begin{array}{l}\text { Vector of planning constraints in the } \mathrm{N} \text {-dimensional solution } \\
\text { space }\end{array}$ & \\
\hline$\Delta x_{i, d}$ & $\begin{array}{l}\text { Relocation of particle } i \text { in generation } \\
d\end{array}$ & $N$-dimensional relocation vector & \\
\hline$p_{\text {best }, i}$ & Previously best position of particle $i$ & Position of so far best-scored plan of particle $i$ & \\
\hline$g_{\text {best }}$ & Global best position & Best-scored position reached so far over all particles & \\
\hline
\end{tabular}

and is one way to limit the relocations. The inertia weight is also used to balance exploration and exploitation of the algorithm. Relatively high values of $\omega$ promote a global search, whereas smaller values are related to a more local search, therefore the inertia weight can be reduced during the course of optimization [34-36]. The second summand is the distance between the actual position of the particle and its best position in the past. This contribution is sometimes called the cognitive component because it is related to the particle's $p_{\text {best }}$, which might be seen as the particle's memory. The third summand is the social component, because it evaluates the distance between the actual position of the individual particle and the overall best position of the whole swarm. The cognitive and the social components are weighted by two vectors of random numbers, $\zeta$ and $\xi$, which introduce the statistical nature of the optimization. $C_{1}$ and $c_{2}$ are optimization constants [24]. An iteration, also called generation in PSO, is finished when all particles have updated their position once. An overview of all parameters is provided in Table 1.

For RT planning each particle is considered one treatment plan containing $N$ different planning constraints. Therefore the position of a plan in the $N$-dimensional solution space is represented by a vector containing the $N$ planning constraints as entries. In a first step each particle has to be initialized randomly with a position and relocation. Then the plan is calculated with our in-house treatment planning system (TPS) Hyperion. Following each plan calculation, the particle fitness is evaluated by a plan quality score (PQS, see Section 2.3). Depending on this PQS the best positions $p_{\text {best }, i}$ and $g_{\text {best }}$ are set and the position update is calculated using Eqs. (4) and (5).

To show the applicability of PSO for automatic VMAT planning this procedure was tested for 10 postoperative prostate cancer cases, with a prescribed equivalent uniform dose (EUD) of 66 Gy to the PTV in addition to two rectum constraints and one bladder constraint. All constraints were serial constraints using gEUD [33]. All cases were retrospectively chosen at random from our clinical database. The swarm for each case contained $I=30$ particles (plans) per generation and the termination criterion was set to $D_{\operatorname{Max}}=100$ generations. The plans were initialized randomly in a range of \pm 5 Gy around a constraint set $G_{j}$ (cf. Eq. (2) in Section 2.1), which was determined by a previous optimization with non-binding rectum and bladder constraints $\left(C_{j}=66 \mathrm{~Gy}\right)$. A summary of all parameters is given in Table 1 .

The PSO and the manipulation of the TPS input files were implemented in Python 2.7.3. A bash-script was used to control the interaction between TPS and PSO.

\subsection{Plan quality score (PQS)}

A prerequisite for PSO is the possibility to measure the quality or fitness of a RT plan to judge different plans and thereby determine the best particle positions $p_{\text {best }, i}(\mathrm{i}=1, \ldots, \mathrm{I})$ and the optimum position of the whole swarm $g_{\text {best }}$. Hence, a PQS is introduced as a measure of particle performance. The overall PQS is defined by a sum of single scores assessing individual dose-volume-histogram (DVH) parameters. For the present prostate cases we used a total of 8 different DVH parameters, which are adapted from the institutional standardized operating procedure (SOP) used for treatment plan acceptance in our department ( $c f$. Table 2). For the PTV EUD, $D_{2 \%}$ and $D_{98 \%}$ are evaluated. The scores are designed to reward adherence and to penalize violation of the planning aims. Fig. 1 schematically shows the three PQS related to PTV parameters.

For OARs the following DVH parameters were evaluated: $D_{2 \%}, V_{60 G y}$ and $V_{40 G y}$ for the rectum and $D_{2 \%}$ and $V_{60 G y}$ for the bladder. The planning aims for rectum and bladder are summarized in Table 2. For further refinement of the PQS, rectum and bladder dose effects $G_{j}$ (cf. Eq. (2) Section 2.1) obtained by the initial plan optimization with nonbinding constraint values ( $C_{j}=66 \mathrm{~Gy}$ ) are used. In principle, it should be possible to decrease the constraint values below these DVH-points for the respective specific patient. Hence an additional, patient individual bend B equaling the resulting patient-specific $G_{j}$ was introduced into the parameter score for OARs ( $c f$. Fig. 2a and b). The

Table 2

Summary of DVH parameters used for Plan Quality Score and plan evaluation. All parameters are taken from our institutional SOP. ( $D_{P}$ prescribed dose 66 Gy).

\begin{tabular}{|c|c|c|c|}
\hline Structure & DVH-parameter & Limit & Explanation \\
\hline \multirow[t]{3}{*}{ PTV } & $D_{98 \%}$ & $>62.7 \mathrm{~Gy}\left(95 \% \mathrm{D}_{\mathrm{P}}\right)$ & Adapted from ICRU Reports 62 and $83[37,38]$ \\
\hline & $D_{2 \%}$ & $<70.6$ Gy $\left(107 \% \mathrm{D}_{\mathrm{P}}\right)$ & \\
\hline & EUD & $=66.0 \mathrm{~Gy}$ & Dose prescription \\
\hline \multirow[t]{3}{*}{ Rectum } & $D_{2 \%}$ & $<66.0$ Gy & ICRU 83 [38] \\
\hline & $V_{40 G y}$ & $<80.0 \%$ & $92 \%$ of patients without Grade $2+$ GI toxicity [39] \\
\hline & $V_{60 G y}$ & $<40.0 \%$ & To prevent moderate/severe toxicity (any endpoint) [40] \\
\hline \multirow[t]{2}{*}{ Bladder } & $D_{2 \%}$ & $<66.0 \mathrm{~Gy}$ & ICRU 83 [38] \\
\hline & $V_{60 G y}$ & $<50.0 \%$ & Institutional standard adapted from QUANTEC $\left(\mathrm{V}_{60 \mathrm{~Gy}}<50 \%\right.$ Grade $3+$ toxicity $)[41]$ \\
\hline
\end{tabular}




\section{PTV Plan Quality Scores}

a) EUD



b) $D_{98 \%}$

c) $D_{2 \%}$
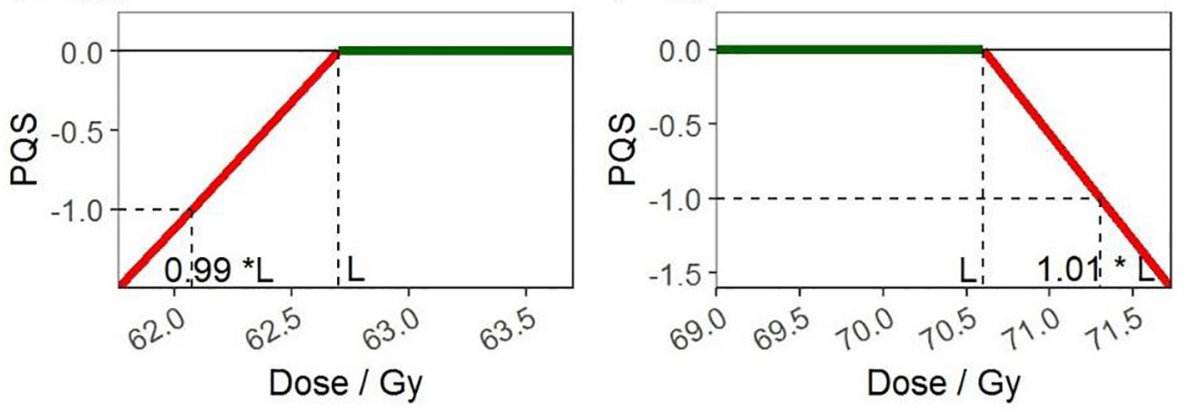

Fig. 1. Schematic illustration of the Plan Quality Scores (PQS) for each of the PTV parameters.

OAR Plan Quality Scores

\section{a) without bend}

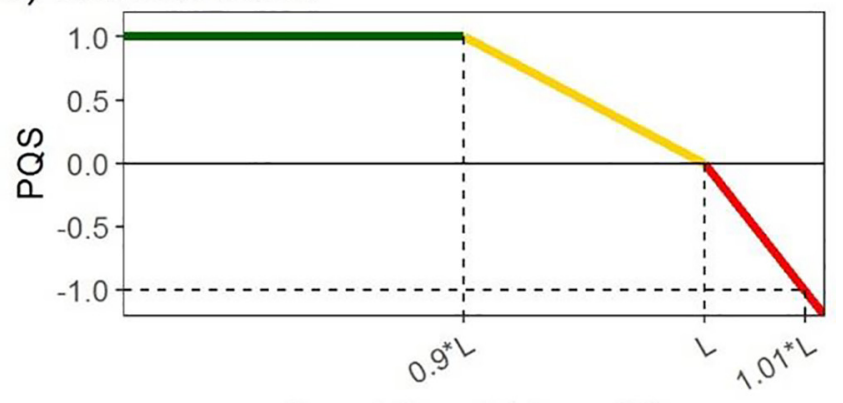

Dose / Gy or Volume / \%

b) with bend



Dose / Gy or Volume / \%

Fig. 2. Schematic illustration of the Plan Quality Scores (PQS) related to the 5 OAR parameters, distinguished into a) PQS without and b) with bend value $B$. Organ specific limits $\mathrm{L}$ are provided in Table 2, bend values B are patient individual. intention here is to guide the optimization below the bend, but to not penalize the exceedance of the bend as hard as the exceedance of the limit. Below the bend a positive score is offered as a reward.

In general, one scoring point can be reached per constraint at maximum, expect for PTV parameters $D_{2 \%}$ and $D_{98 \%}$, which only penalize violation ( $c f$. Fig. $1 \mathrm{~b}$ and c). An extra reward of 5 points each is offered for plans which fulfill all PTV and rectum goals or all PTV and bladder goals, respectively. This means that for plans generated in this study, theoretically a maximum score of $\mathrm{PQS}=16$ points can be reached.

\subsection{PSO plans in comparison to manual plans}

All manual plans were randomly chosen from our clinical database. For manual optimization, the same types of constraints and objectives were used as for the PSO plans, see Section 2.1. The manual plan was always compared to the best scored plan after 100 PSO generations; this plan is denoted as PSO plan hereafter.

The plan quality was evaluated using the same criteria as for the PQS, for a summary see Table 2 . The difference in those parameters between manual and PSO plans was evaluated case-wise. In addition, dose distributions and DVHs were visually inspected and evaluated on a case by case basis.

\subsection{Statistics}

A Wilcoxon signed rank test with Bonferroni correction was carried out using software R (Version 3.5.1) [42], differences between manual and related PSO plans resulting in p-values smaller than 0.05 were considered statistically significant.

\section{Results}

PSO was able to propose plans for all investigated cases. 9 of 10 
a) PC03 Manual plan

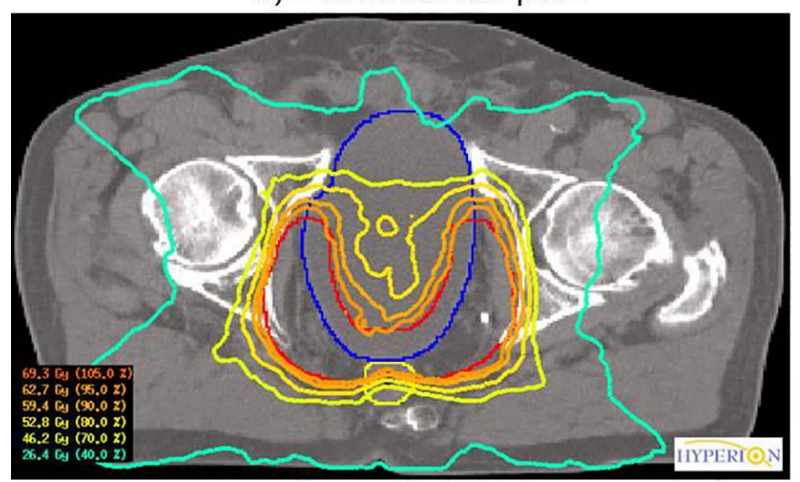

b) PC03 PSO plan



c) PC03 DVH comparison

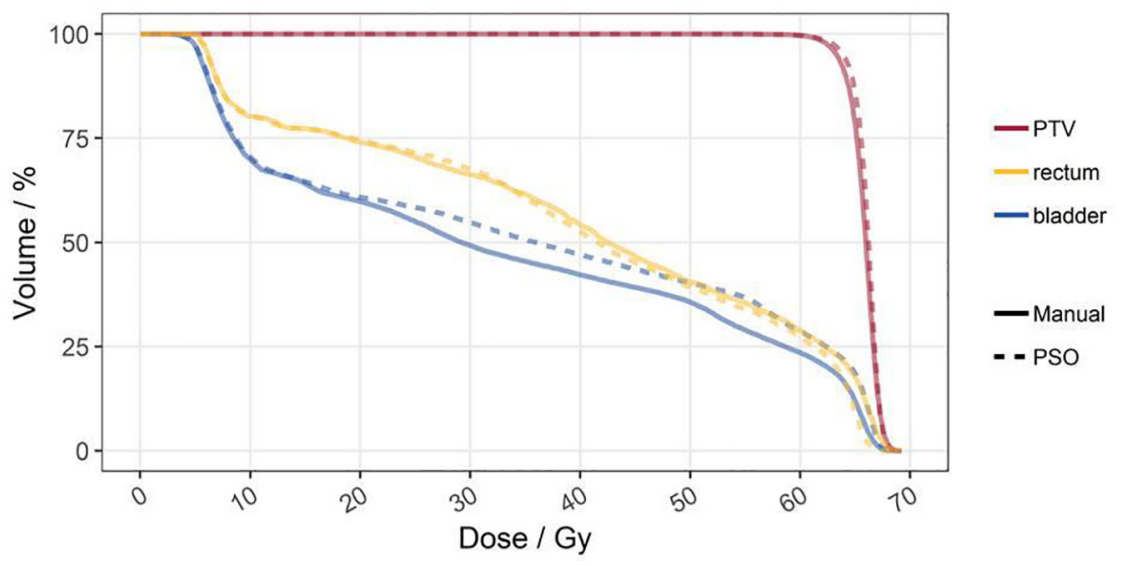

Fig. 3. Case PCO3 as representative example for the 9 successful cases: dose distribution of manual plan (a), PSO plan (b) and DVH comparison (c).

cases were of clinically acceptable quality, one case (PC01) was of inferior plan quality. A comparison of the dose distribution and of the DVHs for two exemplary cases, PC03 and PC01, is provided in Figs. 3 and 4. PC03 is shown as a representative example of the successfully planned cases. The PSO plan suggests a comparable PTV coverage with a PTV EUD of 65.3 Gy in PSO vs. 65.1 Gy, respectively. But the PSO reached better rectum high dose sparing, $D_{2 \%}$ of $66.2 \mathrm{~Gy}$ vs. $67.5 \mathrm{~Gy}$, while allowing for slightly higher bladder doses (see Fig. 3). For PC01 the manual planner decided to exceed the bladder $V_{60 G y}$ restriction $\left(V_{60 G y}<50 \%\right)$ to fulfill the PTV prescription. As PSO adheres to restrictions, due to the penalization in the PQS, it is unable to make such a decision and is therefore unable to propose a clinical acceptable plan in that case (cf. Fig. 4). For detailed case-wise comparisons of DVH parameters for all 10 cases we refer to Table S1 in the supplementary material.

The median (range) in PTV EUD was 65.4 Gy (64.7-66.0) in manual plans and 65.3 Gy $(62.5-65.5, \mathrm{p}=1)$ in PSO plans, respectively. Also the near minimum dose $\left(D_{98 \%}\right)$ is comparable for both plans with 62.9 Gy (61.7-63.6) vs. 62.8 Gy (59.4-63.1, p = 1). However, PSO plans adhere significantly better to the rectum high dose limitation with 66.1 Gy (64.7-66.5) compared to $67.0 \mathrm{~Gy}(66.5-67.5, \mathrm{p}=0.016)$ in manual plans. A summary of all evaluated DVH parameters is provided in Fig. 5 and in the supplementary material (Table 1S).

Fig. 6 provides a DVH comparison for all 9 clinically acceptable plans (PC02-PC10) for PTV and rectum. PC01 was excluded from this analysis as the PSO plan was not clinically reasonable; the DVHs for that case are provided in Fig. 4c. For the DVH comparison in Fig. 6 minimum and maximum volume fraction for each dose bin were plotted, hence all nine individual DVHs fall inside the shaded areas. The area for the PSO plans is much narrower, which indicates that the PSO plans show lower inter-plan variations than the manual plans. Also, the lower interquartile range of the PTV parameters for PSO shown by the boxplots in Fig. 5 encourages the hypothesis that the dispersion of resulting planning parameters is lower. For the rectum, PSO plans propose significantly better sparing in high dose regions, which is also demonstrated by the DVH comparison in Fig. 6. For the volume parameters $V_{40 G y}$ and $V_{60 G y}$ we see a broader range than for $D_{2 \%}$. This reflects the patient individual bends in the PQSs for these parameters, which is also visible in the wider boxes in Fig. 5.

Fig. 7 summarizes individual and total PQS for all 10 cases. It supports the findings that PTV EUDs are comparable for manual and PSO plans in most cases. For the rectum $D_{2} \%$ PSO plans scored always better than manual plans which lead to better rectum high dose sparing. In addition rectum $V_{60 G y}$ reached a better sparing in PSO plans than in manual plans, which is visible in the higher scores in Fig. 7. But for most cases PSO plans scored slightly worse than manual plans in rectum $V_{40 G y}$. For 8 of 10 cases, the total PQS is higher in PSO plans compared to manual plans, which was to be expected as the PSO uses the PQS directly as guidance for the optimization, whereas a human planner knows in principle the same limits, but he does not get a direct feedback about the PQS during the trial-and-error planning process. The supplementary Fig. S1 provides an additional summary of the total PQS for all patients.

\section{Discussion}

Our in-house developed TPS Hyperion uses a constrained optimization to generate a pareto-optimal plan. Depending on the used planning constraints this may be a clinically acceptable plan or not. To overcome that problem, manual planners need to try out different combinations of planning constraints to find an appropriate plan for each individual patient. This trial-and-error process could be seen as a 
a) PC01 Manual plan

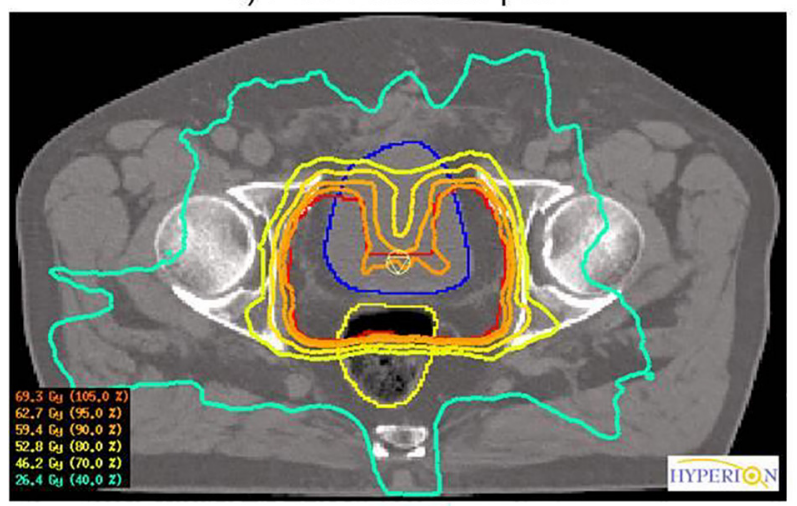

b) PC01 PSO plan

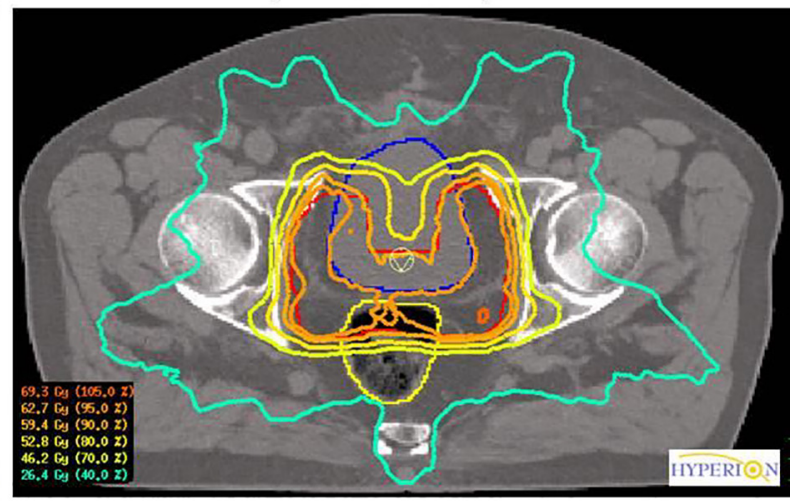

c) PC01 DVH comparison




acceptable plan due to the bladder $V_{60 G y}<50 \%$ restriction ( $c f$. arrow).

manual exploration of the pareto-surface. But this exploration is inefficient and might be biased by the experience of the planner. Therefore, we propose an automatic planning based on PSO, which can also be interpreted as an automatic navigation on the pareto-surface, with the aim to explore the pareto-surface efficiently and select the optimal, clinically acceptable plan for the patient automatically. Navigation on the pareto-surface was already proposed by Craft et al. $[43,44]$, they approximate the pareto-surface by a linear-combination of a few pre-calculated pareto-optimal plans. Then a manual planner can explore the pareto-surface and has to decide for one plan. The main
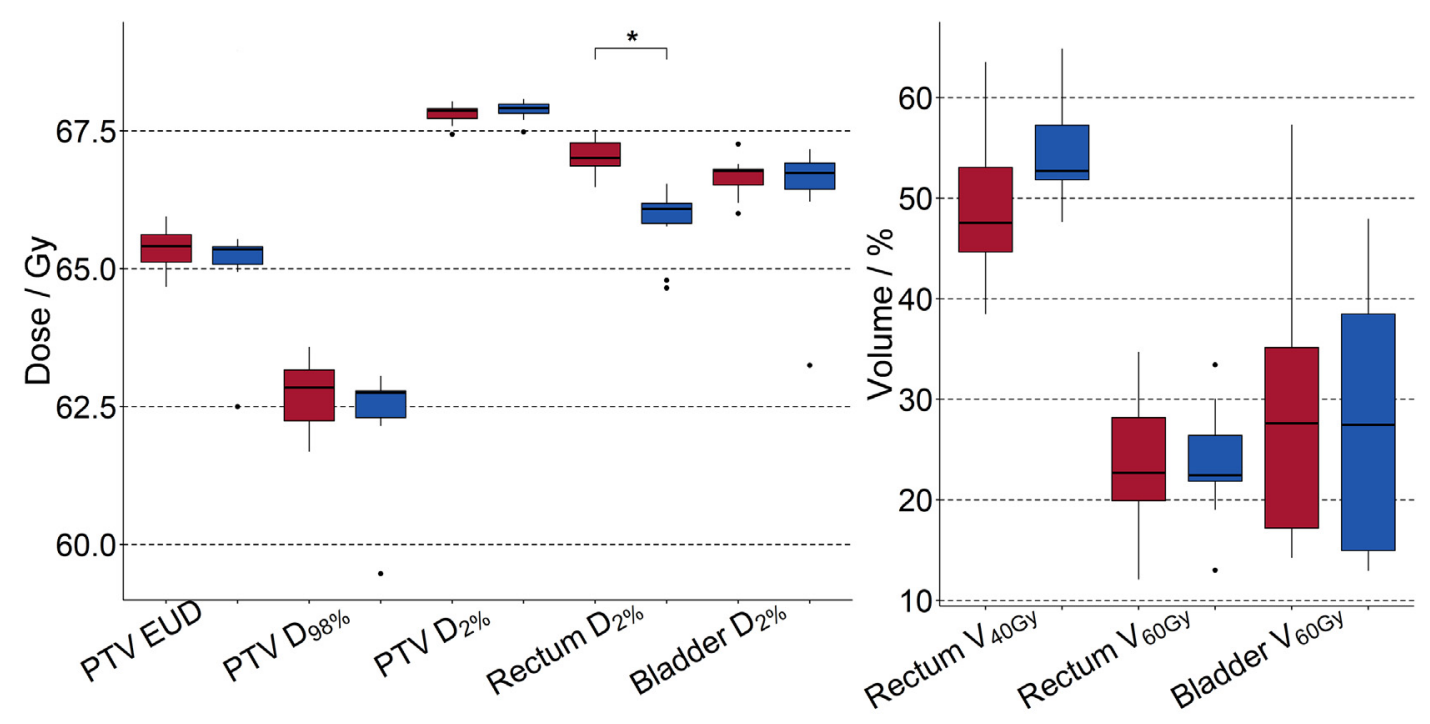

manual m PSO

Fig. 5. Boxplots showing the distribution of DVH-parameters for the 10 cases $\left({ }^{*} \mathrm{p}<0.05\right.$, ${ }^{* *} \mathrm{p}<0.01$ ). 
a) PTV

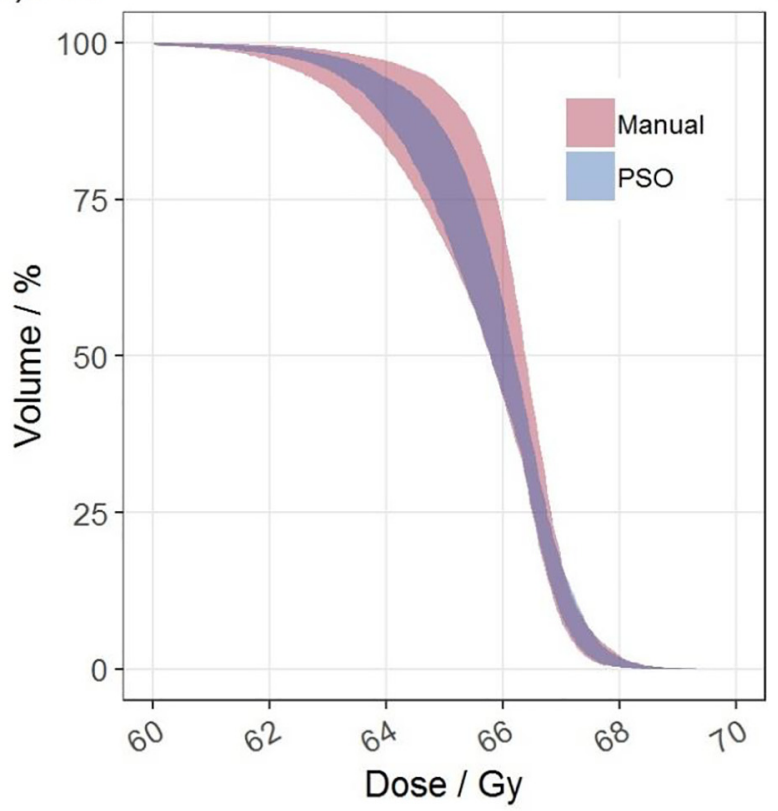

b) Rectum

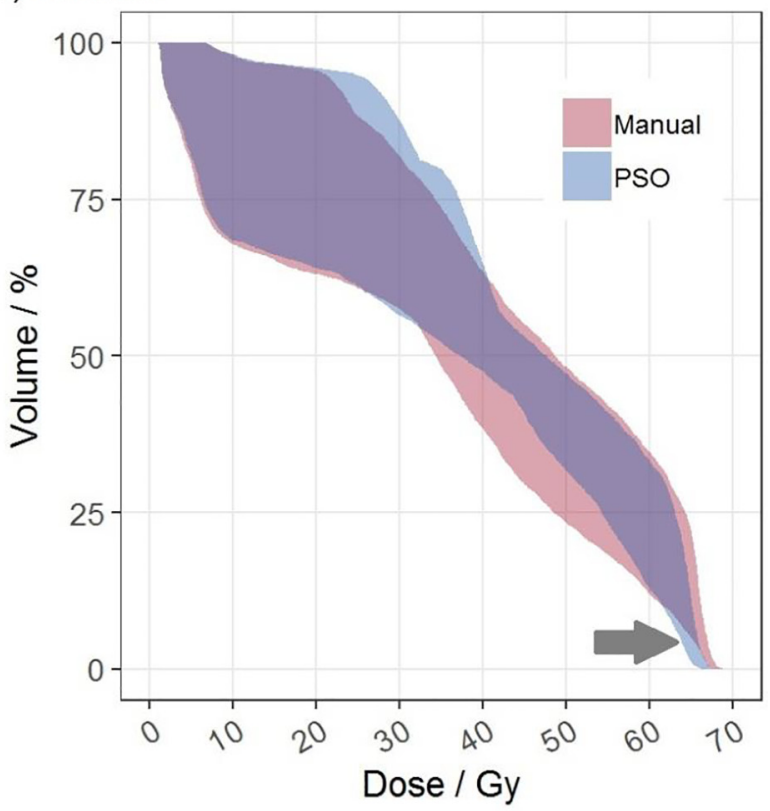

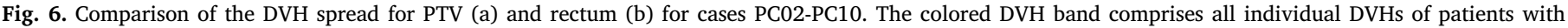
clinically acceptable PSO plans (PC02-PC10). The arrow in b) highlights the high dose region, where manual and PSO plans are clearly separated.

difference to our approach is that we explore the pareto-surface automatically, but therefore we need to calculate a large number of paretooptimal plans, hence our approach is much more expensive in terms of computational costs. At the moment we need around 3-5 min per plan on our server with 18 Intel $^{\circledR}$ Xeon ${ }^{\circledR}$ Processors X5650. For a swarm of 30 particles executing 100 generations this sums up to a total computation time of $150-250 \mathrm{~h}$, at the moment this would impede clinical application. But so far the PSO was not optimized for computation time. As the particle updates in each generation can be determined mutually independent from each other, a straightforward way to speed up the optimization routine would be to calculate the new particle positions in parallel. Another option to speed up the plan computation would be


Rectum $V_{40 G y}$
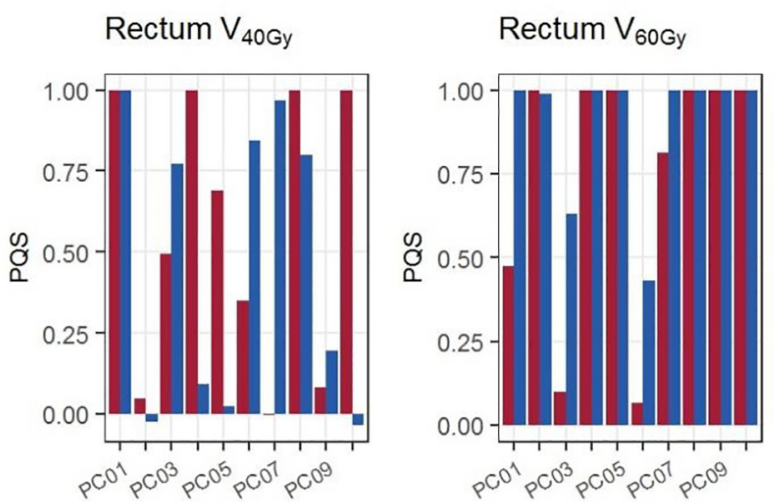
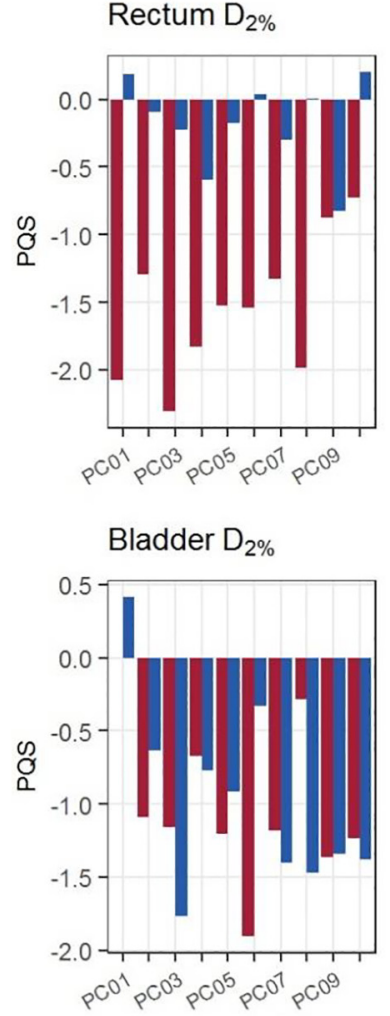

total


Fig. 7. Overview of the individual scores for all 10 cases for manual and PSO plans. PTV $D_{2 \%}$ is not presented as all cases fulfilled this parameter (PQS $=0$ ), bladder $V_{60 G y}$ is not presented as all cases obtained PQS $=1$, except for PC01:-14.60 Manual and 0.41 PSO. 
GPU computing, which is not yet used in our TPS. And also the PSO implementation needs improvement, especially the choice of the inertia weight $\omega$ is crucial for the convergence ability and consequentially for the overall speed of the PSO $[36,45]$.

Automatic planning aims to overcome different challenges of manual planning. In contrast to manual trial-and-error RT planning PSO is objective, even unlikely positions are explored, and reproducible. But the termination criterion needs further improvement, by implementing e.g. a convergence criterion to the PSO. Fig. $2 \mathrm{~S}$ in the supplementary material visualizes the convergence of the PSO as a function of the generation number. Also the PSO optimization parameters ( $c f$. Table 1) should be investigated onwards. A further issue is the lack of tools to independently measure the quality of a treatment plan, i.e. to perform an objective inter- or intra-patient plan comparison. Human planners are normally empirically affected in their choices and do not have a clearly defined quality measure in mind. In PSO the PQS is used to evaluate treatment plans and to guide the optimization. Therefore, all cases that refer to the same PQS also refer to the same well defined standard and should therefore reduce inter-patient variation. Nevertheless, the plan quality score is able to account for individual patient conditions, i.e. if a better OAR sparing is possible without compromising PTV the PQS prefers that compromise. And the individual PQS could be customized for DVH parameters which are highly related to patient anatomy, such as the volume parameters $V_{40 G y}$ and $V_{60 G y}$ for the rectum.

In one case (PC01) PSO was unable to propose a clinically acceptable plan due to the patient anatomy. In manual planning the decision was made to exceed the requested bladder dose to ensure PTV dose. As the PQS is designed for a common clinical situation and is thus unable to reflect this compromise, the PSO compromised the PTV dose (EUD $=62.5 \mathrm{~Gy}$ ) to ensure bladder sparing. The decision to sacrifice the OAR sparing for PTV dose in this case is in the clinician's responsibility and can only be taken individually for each patient depending on the clinical situation. Hence, a discussion with the planner followed by manual planning would be still necessary in such cases.

Three conceptually different approaches have been proposed so far for automatic RT planning: atlas, deep learning and template-based approaches. Atlas based concepts [11-14] and deep learning [19-21] concepts rely on databases with plans of previously treated patients. Depending on the quality of that database the quality of the automatic plan will differ. Deep learning has not yet been used to plan VMAT treatments [19-21]. Pinnacle ${ }^{3}$ AutoPlanning, based on regional optimization [18] and Erasmus iCylcle [15,16] are template-based approaches. iCycle uses a so called wishlist to formulate the planning goals, this wishlist has a categorical order of hard and soft constraints related to planning volumes and OARs, concerning the priority of the structures. This order is fixed for all patients, but the constraint value is automatically altered patient specific.

For automatic planning with Pinnacle ${ }^{3}$ AutoPlanning and Erasmus iCycle gains over all regions of the DVH were reported [4,46-48], whereas in our approach only a gain for high-dose regions in the rectum DVH were shown, which reflects the conceptual difference of our strategy. The in-house TPS used in this study provides for all planning problems pareto-optimal plans. Nevertheless, a pareto-optimal plan yields a clinically favorable plan, if competing constraints are well balanced and if the selected constraints reflect and control the clinical interest. As the selected constraint types were not changed between manual and PSO plans and all plans were generated by TPS Hyperion, and are therefore considered pareto-optimal, a gain in all DVH regions at the same time is not to be expected. The fact that we were able to show gains for the rectum high-dose region at the cost of a slightly increased bladder dose, which still adheres to institutional presets, is due to the definition of the PQS. In principle other compromises might have been possible, depending on the definition and implementation of the PQS.

In our PSO approach the PQS guides the optimization. With the individual PQS for different structures it is possible to prioritize as well, by defining different maximum values. But a less prioritized OAR could outweigh a high prioritized OAR if the dose sparing is clearly better, because better dose sparing means increasing PQS. On the other hand, this flexibility in PQS makes it difficult to design it robustly. Here we presented a relatively simple PQS specifically optimized for postoperative-prostate cases. The design of the PQS is treatment site and treatment intent specific, even more complex prostate treatments would require a refined $\mathrm{PQS}$ integrating additional OARs, such as urethra, penile bulb and femoral heads. By now the PQS solely comprised DVH parameters for PTV and two major OARs. DVH parameters are clinically well-known measures of plan quality and were therefore chosen as basis for the PQS, but in principle it should be possible to integrate further characteristics such as machine parameters or complex tumor control probability (TCP) and normal tissue complication probability (NTCP) models in the future. Yang et al. proposed to use PSO to set the weighting factors in IMRT optimization [31]. This is similar to our approach, but a main difference is, that we do not use PSO as the IMRT/ VMAT optimizer but as a second optimization, with the intention to explore the pareto-surface automatically. Therefore, our approach should be of general applicability for all TPS which generate paretooptimal plans.

\section{Conclusion}

In this study, PSO was successfully implemented and used for VMAT treatment planning of postoperative prostate cancer RT with similar plan quality compared to manually optimized VMAT plans. Hence, automatic treatment planning using PSO is technically feasible and highly promising for future clinical applications. PSO relies on a quality measure to compare different treatment plans. For this purpose, a dedicated plan quality score (PQS) was developed in this work. This PQS needs further refinement and optimization before PSO can be used clinically for automatic treatment planning. Further planning studies and comparative trials are needed to prove the clinical applicability of this approach.

\section{Acknowledgments}

The project received funding by the Medical Faculty of the University of Tübingen, Germany, Fortüne Grant-No. 2414-0-0.

We further thank Markus Alber for providing us with the research TPS Hyperion.

\section{Conflicts of Interest}

DZ and DT have research collaborations with Elekta AB (Sweden), Philips (The Netherlands) and Siemens Healthineers (Germany). All other authors have no relevant conflicts of interest to disclose.

\section{Appendix A. Supplementary data}

Supplementary data to this article can be found online at https:// doi.org/10.1016/j.ejmp.2019.12.007.

\section{References}

[1] Barth NH. An inverse problem in radiation therapy. Int J Radiat Oncol Biol Phys 1990;18:425-31. https://doi.org/10.1016/0360-3016(90)90111-V.

[2] Oelfke U, Bortfeld T. Inverse planning for X-ray rotation therapy: a general solution of the inverse problem. Phys Med Biol 1999:1089-104. https://doi.org/10.1088/ 0031-9155/44/4/019.

[3] Alber M. Normal tissue dose-effect models in biological dose optimisation. Zeitschrift Für Medizinische Physik 2008;18:102-10. https://doi.org/10.1016/j. zemedi.2007.08.002.

[4] Buschmann M, Sharfo AWM, Penninkhof J, Seppenwoolde Y, Goldner G, Georg D, et al. Automated volumetric modulated arc therapy planning for whole pelvic prostate radiotherapy, Strahlentherapie Und. Onkologie 2017:333-42. https://doi. 
org/10.1007/s00066-017-1246-2

[5] Court LE, Kisling K, McCarroll R, Zhang L, Yang J, Simonds H, et al. J Visual Exp 2018:e57411.

[6] Krayenbuehl J, Zamburlini M, Ghandour S, Pachoud M, Tol S, Guckenberger M, et al. Planning comparison of five automated treatment planning solutions for locally advanced head and neck cancer. Radiat Oncol 2018;13. https://doi.org/10. 1016/S0167-8140(18)30823-5.

[7] Sharfo AWM, Stieler F, Kupfer O, Heijmen BJM, Dirkx MLP, Breedveld S, et al. Automated VMAT planning for postoperative adjuvant treatment of advanced gastric cancer. Radiat Oncol 2018;13:74. https://doi.org/10.1186/s13014-018-1032-z.

[8] Vanderstraeten B, Goddeeris B, Vandecasteele K, van Eijkeren M, de Wagter C, Lievens Y. Automated Instead of Manual Treatment Planning? A Plan Comparison Based on Dose-Volume Statistics and Clinical Preference. Int J Radiat Oncol Biol Phys 2018;102:443-50.

[9] Wang J, Chen Z, Li W, Qian W, Wang X, Hu W. A new strategy for volumetricmodulated arc therapy planning using AutoPlanning based multicriteria optimization for nasopharyngeal carcinoma. Radiat Oncol 2018;13:1-10. https://doi.org/ 10.1186/s13014-018-1042-x.

[10] Rossi L, Sharfo AW, Aluwini S, Dirkx M, Breedveld S, Heijmen B. First fully automated planning solution for robotic radiosurgery - comparison with automatically planned volumetric arc therapy for prostate cancer. Acta Oncol 2018:1-9. https:// doi.org/10.1080/0284186X.2018.1479068.

[11] Li N, Zarepisheh M, Uribe-Sanchez A, Moore K, Tian Z, Zhen X, et al. Automatic treatment plan re-optimization for adaptive radiotherapy guided with the initial plan DVHs. Phys Med Biol 2013;58:8725-38. https://doi.org/10.1088/0031-9155/ 58/24/8725.

[12] Zarepisheh M, Long T, Li N, Tian Z, Romeijn HE, Jia X, et al. A DVH-guided IMRT optimization algorithm for automatic treatment planning and adaptive radiotherapy replanning. Med Phys 2014;41. https://doi.org/10.1118/1.4875700.

[13] McIntosh C, Welch M, McNiven A, Jaffray DA, Purdie TG. Fully automated treatment planning for head and neck radiotherapy using voxel-based dose prediction and dose mimicking method. Phys Med Biol 2017;62:5926. https://doi.org/10. 1088/1361-6560/aa71f8.

[14] McIntosh C, Purdie TG. Voxel-based dose prediction with multi-patient atlas selection for automated radiotherapy treatment planning. Phys Med Biol 2017;62:415. https://doi.org/10.1088/1361-6560/62/2/415.

[15] Breedveld S, Storchi PRM, Keijzer M, Heemink AW, Heijmen BJM. A novel approach to multi-criteria inverse planning for IMRT. Phys Med Biol 2007;52:6339-53. https://doi.org/10.1088/0031-9155/52/20/016.

[16] Voet PWJ, Breedveld S, Dirkx MLP, Levendag PC, Heijmen BJM. Integrated multicriterial optimization of beam angles and intensity profiles for coplanar and noncoplanar head and neck IMRT and implications for VMAT. Med Phys 2012;39:4858-65. https://doi.org/10.1118/1.4736803.

[17] Winkel D, Bol GH, van Asselen B, Hes J, Scholten V, Kerkmeijer LGW, et al Development and clinical introduction of automated radiotherapy treatment planning for prostate cancer. Phys Med Biol 2016;61:8587-95. https://doi.org/10. 1088/1361-6560/61/24/8587.

[18] Xhaferllari I, Wong E, Bzdusek K, Lock M, Chen J. Automated IMRT planning with regional optimization using planning scripts. J Appl Clin Med Phys 2013;14:4052. https://doi.org/10.1120/jacmp.v14i1.4052.

[19] Fan J, Wang J, Chen Z, Hu C, Zhang Z, Hu W. Automatic treatment planning based on three-dimensional dose distribution predicted from deep learning technique. Med Phys 2019;46:370-81. https://doi.org/10.1002/mp.13271.

[20] Chen X, Men K, Li Y, Yi J, Dai J. A feasibility study on an automated method to generate patient-specific dose distributions for radiotherapy using deep learning. Med Phys 2019;46:56-64. https://doi.org/10.1002/mp.13262.

[21] Kearney V, Chan JW, Haaf S, Descovich M, Solberg TD. DoseNet: a volumetric dose prediction algorithm using 3D fully-convolutional neural networks. Phys Med Biol 2018;63:235022https://doi.org/10.1088/1361-6560/aaef74.

[22] Nelms BE, Robinson G, Markham J, Velasco K, Boyd S, Narayan S, et al. Variation in external beam treatment plan quality: An inter-institutional study of palnners and planning systems. Pract Radiat Oncol 2012;2:296-305. https://doi.org/10.1016/j. prro.2011.11.012.

[23] Ventura T, Lopes M do C, Ferreira BC, Khouri L. SPIDERplan: a tool to support decision-making in radiation therapy treatment plan assessment. Rep Pract Oncol Radiother 2016;21:508-16. https://doi.org/10.1016/j.rpor.2016.07.002.

[24] Kennedy J, Eberhart R, Particle swarm optimization, Neural Networks, 1995. Proceedings., IEEE International Conference On. 4 (1995) 1942-1948, doi: 10. 1109/ICNN.1995.488968.

[25] Lee CH, Shih KS, Hsu CC, Cho T. Simulation-based particle swarm optimization and mechanical validation of screw position and number for the fixation stability of a femoral locking compression plate. Med Eng Phys 2014;36:57-64. https://doi.org/ 10.1016/j.medengphy.2013.09.005

[26] Wisittipanich W, Hengmeechai P. Truck scheduling in multi-door cross docking terminal by modified particle swarm optimization. Comput Ind Eng 2017;113:793-802. https://doi.org/10.1016/j.cie.2017.01.004.

[27] Li Y, Yao D, Yao J, Chen W. A particle swarm optimization algorithm for beam angle selection in intensity-modulated radiotherapy planning. Phys Med Biol 2005;50:3491-514. https://doi.org/10.1088/0031-9155/50/15/002.

[28] Riofrio D, Luan S, Zhou J, Ma L. Particle swarm optimization for radiation therapy planning. BCB 2015 - 6th ACM Conference on Bioinformatics, Computational Biology, and Health Informatics. 2015. p. 250-7. doi: 10.1145/2808719.2808745.

[29] Modiri A, Gu X, Hagan A, Bland R, Iyengar P, Timmerman R, et al. Inverse 4D conformal planning for lung SBRT using particle swarm optimization. Phys Med Biol 2016;61. https://doi.org/10.1088/0031-9155/61/16/6181.

[30] Modiri A, Gu X, Hagan AM, Sawant A. Radiotherapy Planning Using an Improved Search Strategy in Particle Swarm Optimization. IEEE Trans Biomed Eng 2017;64:980-9. https://doi.org/10.1109/TBME.2016.2585114.

[31] Yang J, Zhang P, Zhang L, Shu H, Li B, Gui Z. Particle swarm optimizer for weighting factor selection in intensity-modulated radiation therapy optimization algorithms. Physica Med 2017;33:136-45. https://doi.org/10.1016/j.ejmp.2016. 12.021.

[32] Alber M, Reemtsen R. Intensity modulated radiotherapy treatment planning by use of a barrier-penalty multiplier method. Optimiz Methods Software 2007;22:391-411. https://doi.org/10.1080/10556780600604940.

[33] Niemierko A. A generalized Concept of Equivalent Uniform Dose (EUD). Med Phys 1999 (accessed September 27, 2019). https://www.aapm.org/meetings/99AM/ pdf/2682-56794.pdf.

[34] Shi Y, Eberhart R. A Modified Particle Swarm Optimizer. Proceedings of the 1998 IEEE International Conference on Evolutionary Computation. IEEE World Congress on Computational Intelligence. 1998. p. 69-73. doi: 10.1109/ICEC.1998.699146.

[35] Shi Y, Eberhart R. Empirical study of particle swarm optimization. Proceedings of the 1999 Congress on Evolutionary Computation. 1999. p. 1945-50. doi: 10.1109/ CEC.1999.785511.

[36] Nickabadi A, Ebadzadeh MM, Safabakhsh R. A novel particle swarm optimization algorithm with adaptive inertia weight. Appl Soft Comput J 2011;11:3658-70. https://doi.org/10.1016/j.asoc.2011.01.037.

[37] ICRU. Report 62: Prescribing, Recording and Reporting Photon Beam Therapy (Supplement to ICRU Report 50). J ICRU 1999:0s32.

[38] ICRU. Report 83: Prescribing, Recording, and Reporting Photon-Beam IntensityModulated Radiation Therapy (IMRT). J ICRU 2010;10.

[39] Pederson AW, Fricano J, Correa D, Pelizzari CA, Liauw SL. Late toxicity after intensity-modulated radiation therapy for localized prostate cancer: An exploration of dose-volume histogram parameters to limit genitourinary and gastrointestinal toxicity. Int J Radiat Oncol Biol Phys 2012;82:235-41. https://doi.org/10.1016/j. ijrobp.2010.09.058.

[40] Gulliford SL, Foo K, Morgan RC, Aird EG, Bidmead AM, Critchley H, et al. DoseVolume Constraints to Reduce Rectal Side Effects From Prostate Radiotherapy: Evidence From MRC RT01 Trial ISRCTN 47772397. Int J Radiat Oncol Biol Phys 2010;76:747-54. https://doi.org/10.1016/j.ijrobp.2009.02.025.

[41] Viswanathan AN, Yorke ED, Marks LB, Eifel PJ, Shipley WU. Radiation DoseVolume Effects of the Urinary Bladder. Int J Radiat Oncol Biol Phys 2010;76:116-22. https://doi.org/10.1016/j.ijrobp.2009.02.090.

[42] R Core Team (2018), R: A language and Enviorment for Statistical Computing, R Foundation for Statistical Computing, Vienna, Austria, URL https://www.R-project. org/, (n.d.). https://www.r-project.org/.

[43] Craft DL, Halabi TF, Shih HA, Bortfeld TR. Approximating convex Pareto surfaces in multiobjective radiotherapy planning. Med Phys 2006;33:3399-407. https://doi. org $/ 10.1118 / 1.2335486$.

[44] Craft D, Monz M. Simultaneous navigation of multiple Pareto surfaces, with an application to multicriteria IMRT planning with multiple beam angle configurations. Med Phys 2010;37:736-41. https://doi.org/10.1118/1.3292636.

[45] Bansal JC, Singh PK, Saraswat M, Verma A, Jadon SS, Abraham A. Inertia Weight Strategies in Particle Swarm Optimization. In: Abrahameditor. Third World Congress on Nature and Biologically Inspired Computing IEEE; 2011. p. 633-40. https://doi.org/10.1109/NaBIC.2011.6089659.

[46] McConnell KA, Marston T, Zehren BE, Lirani A, Stanley DN, Bishop A, et al. Dosimetric evaluation of pinnacle's automated treatment planning software to manually planned treatments. Technol Cancer Res Treat 2018;17:1-7. https://doi. org $/ 10.1177 / 1533033818780064$.

[47] Hansen CR, Bertelsen A, Hazell I, Zukauskaite R, Gyldenkerne N, Johansen J, et al. Automatic treatment planning improves the clinical quality of head and neck cancer treatment plans. Clin Transl Radiat Oncol 2016;1:2-8. https://doi.org/10.1016/j. ctro.2016.08.001.

[48] Sharfo AWM, Dirkx MLP, Bijman RG, Schillemans W, Breedveld S, Aluwini S, et al. Late toxicity in the randomized multicenter HYPRO trial for prostate cancer analyzed with automated treatment planning. Radiother Oncol 2018;128:349-56. https://doi.org/10.1016/j.radonc.2018.05.028. 